\begin{tabular}{|l|l|}
\hline $\begin{array}{l}\text { 2. To: (Receiving Organization) } \\
\text { Characterization Project Operations }\end{array}$ & $\begin{array}{l}\text { 3. From: (Originating Organization) } \\
\text { Characterization Field Engineering }\end{array}$ \\
\hline 5. Proj./Prog./Dept./Div.: & $\begin{array}{l}\text { 6. Design Authority/Design Agent/Cog. Engr.: } \\
\text { Characterization Project }\end{array}$ \\
\hline
\end{tabular}

Characterization Project

8. Originator Remarks:

Distributed for approval and release.

\begin{tabular}{|c|} 
4. Related EDT No.: \\
N/A \\
$\begin{array}{r}\text { 7. Purchase Order No.: } \\
\text { N/A }\end{array}$ \\
9. Equip./Component No.: \\
N/A \\
\hline $\begin{array}{r}\text { 10. System/B/dg./Facility: } \\
200 G\end{array}$
\end{tabular}

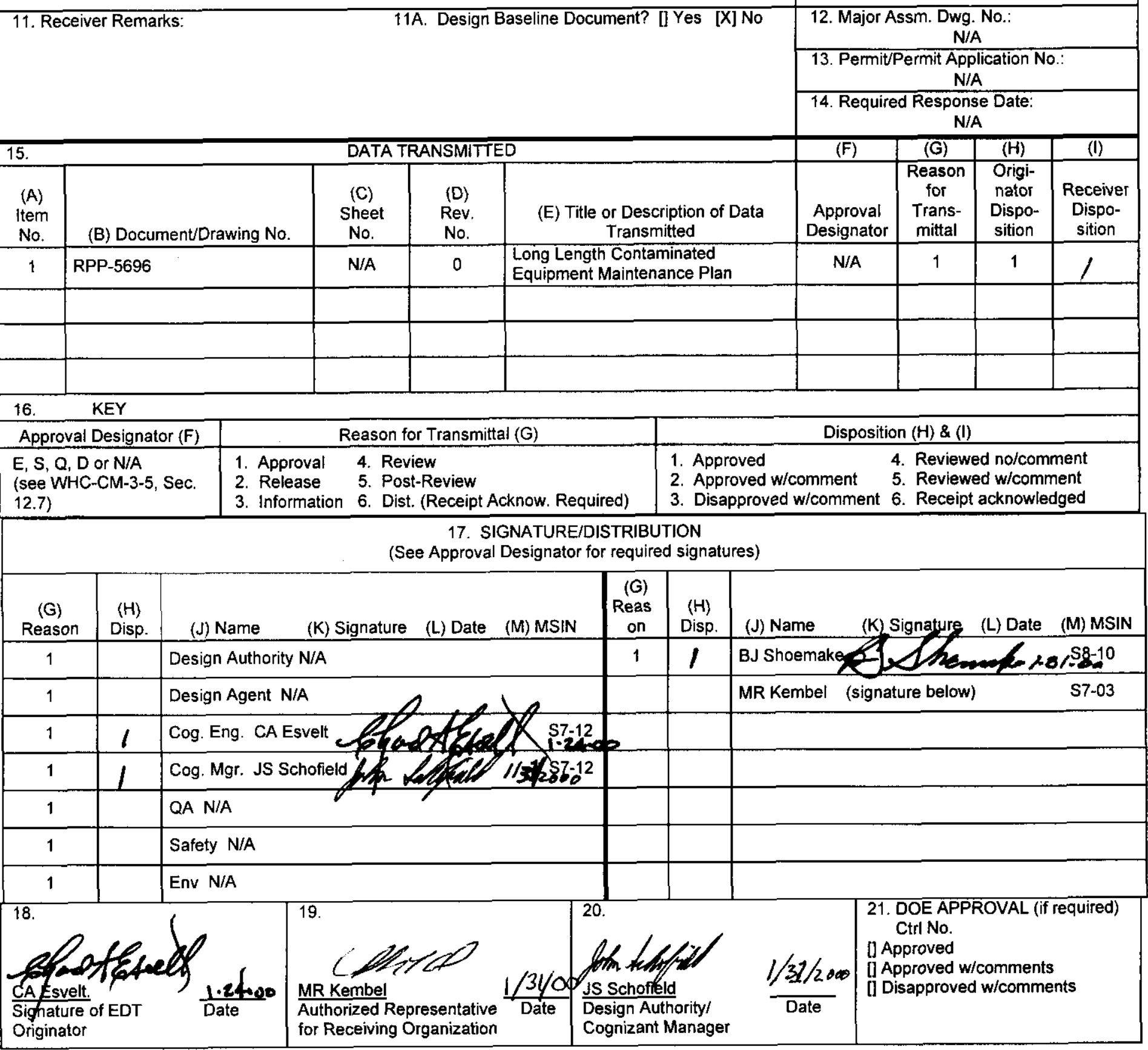




\section{Long Length Contaminated Equipment Maintenance Plan}

\section{C.A. Esvelt}

Prepared by $\mathrm{CH} 2 \mathrm{Mhill}$ Hanford Group Inc.

Richland, WA 99352

U.S. Department of Energy Contract DE-AC06-96RL13200

EDT: 627885

Org Code: 74900

B\&R Code: EW3120074
UC: 2070

Charge Code: 110337

Total Pages:

Key Words: Long Length Contaminated Equipment, LLCE, Maintenance

Abstract: This maintenance plan contains the listing of maintenance activities and the determined frequency for the LLCE trailers for the River Protection Project (RPP).

TRADEMARK DISCLAIMER. Reference herein to any specific commercial product, process, or service by trade name, trademark, manufacturer, or otherwise, does not necessarily constitute or imply its endorsement, recommendation, or favoring by the United States Government or any agency thereof or its contractors or subcontractors.

Printed in the United States of America. To obtain copies of this document, contact: Document Control Services, P.O. Box 950, Mailstop H6-08, Richland WA 99352, Phone (509) 372-2420; Fax (509) 376-4989.
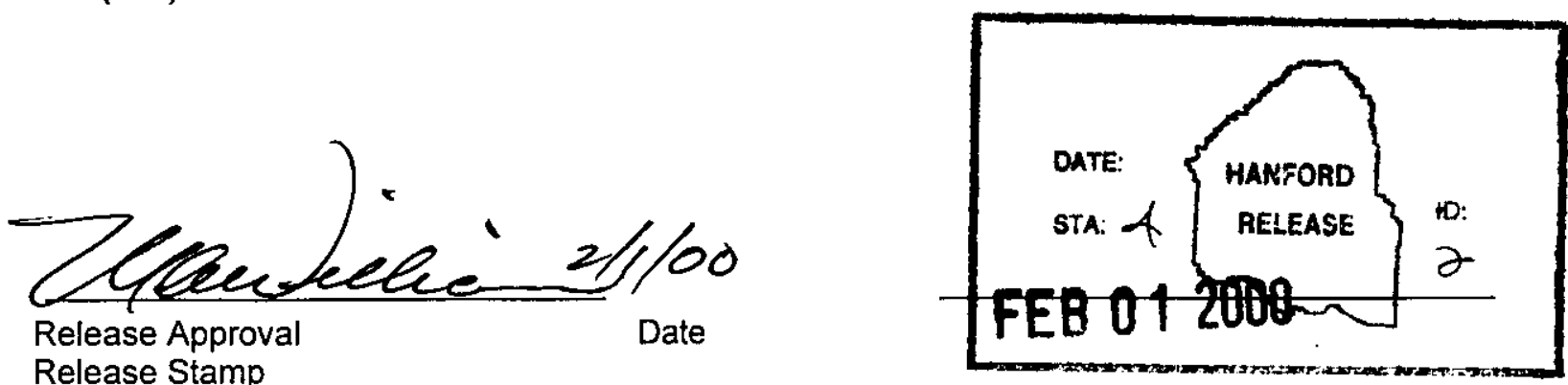

Release Stamp

A-6400-073 (01/97) GEF321

Approved for Public Release 


\section{PURPOSE}

The purpose of this document is to provide the maintenance requirements of the Long Length Contaminated Equipment (LLCE) trailers and provide a basis for the maintenance frequencies selected.

\section{SCOPE}

This document is applicable to the LLCE Receiver trailer and Transport trailer assembled by Mobilized Systems Inc. (MSI). Equipment used in conjunction with, or in support of, these trailers is not included. This document does not provide the maintenance requirements for checkout and startup of the equipment following the extended lay-up status which began in the mid 1990s. These requirements will be specified in other documentation.

\section{BACKGROUND}

The LLCE disposal system is comprised of equipment and processes for retrieving, packaging, and transporting long length equipment from Hanford waste tanks. The LLCE transport system will be used during the LLCE disposal process to handle and move long length radioactive contaminated equipment into high density plastic burial containers. The transportation system consists of two trailers: a receiver trailer to receiver equipment from the waste tanks and a transport trailer to transport the burial container to the disposal facility.

Since 1996, these trailers have been parked in the laydown yard, north of $2704 \mathrm{HV}$, exposed to the weather. They have never been put into service.

MSI has provided 'product literature sheets' (cutsheets) for the components used in the assembly of the trailers. This information is provided in Hanford site vendor information file (VI) 22809.

However, there has been no definitive maintenance plan written for these trailers. This document will discuss and list the maintenance required for the LLCE transport system.

Three general types of maintenance activities have been identified within the vendor documentation for the LLCE trailers: pre-use maintenance, preventative maintenance, and longterm storage activities.

Pre-use maintenance activities are tasks that will be scheduled and performed on operating equipment and systems prior to each campaign. Pre-use maintenance activities include lubrication, adjustments, and inspections.

Preventative maintenance includes those activities necessary to evaluate the condition of equipment, maintain equipment in the operational mode, prevent equipment breakdown, and identify corrective maintenance. Preventative maintenance activities include periodic lubrication, adjustments, calibrations, and inspections. Any calibration of equipment shall be included in the preventative maintenance schedule. The frequency selected for preventative maintenance tasks is based primarily on the estimated operating time of the LLCE trailers.

Long term maintenance activities are those tasks used to prevent deterioration of equipment during long term storage when equipment is not used.

Implementation of these recommended maintenance activities will ensure the trailers are maintained in an operational condition. 


\section{DISCUSSION}

Appendix $A$ is a compilation of all available vendor information, maintenance recommendations, and information from previous documents produced for the LLCETS. Appendix A includes all vendor recommended maintenance requirements and frequencies for the trailers' main components.

The information in Appendix $A$ is not in a form which can be readily usable for setting up a maintenance program within RPP for the LLCETS. Some vendor recommended frequencies are based upon of time, others are based upon time of operation or distance traveled. The vendor recommendations are assumed to be based upon 160 hours per month of operation. This is a much higher usage rate than will actually occur. A more realistic assessment of LLCETS usage was used in preparation of this document. This document was based on the assumption that the equipment would be utilized for one LLCE removal per month, and that both the receiver and the transport trailer would be operated for a maximum of 16 hours per LLCE removal.

This document divides recommended maintenance activities into four categories; Pre-use, Preventative, Upon Request and Long-Term Storage. Separation of the maintenance tasks into the four categories was determined by the cognizant engineer for the LLCETS. This decision was based on vendor component information, craft knowledge and recommendations, discussions with MSI, and engineering judgement.

Pre-use maintenance activities are those activities to be performed prior to each trailer deployment. Appendix $B$ lists the pre-use maintenance inspections. This includes all the items from Appendix A which are recommended to be performed on a daily or other short-term basis, or that Engineering recommends being completed to ensure safe equipment operation. The items listed in Appendix B under 'Pre-use' will be performed as an integral prerequisite step of the operational work package. At this point, the operational work package has not been developed, however this work package will have numerous prerequisite steps that must be completed before field deployment.

Preventative maintenance includes all routine equipment calibrations, lubrications, leak checks, and preventive maintenance activities. A single preventative maintenance frequency of six months was selected to provide one outage period rather than having numerous small outages. Appendix $C$ lists the preventative maintenance requirements for the LLCETS. Items listed in Appendix A with a recommended frequency of less than 6 months or 500 hours of operation are included in Appendix C. Assuming one LLCE removal per month and 16 hours of operation for each trailer per LLCE removal indicates each trailer will be operated about 100 hours in a six month period. This estimated operating time in a six month period will be about $60 \%$ of what the vendor assumed would occur in one month. Therefore, performing maintenance every six months on items recommended by the vendor to be maintained on a one to six month or 500 hours of operation basis is acceptable. Those items recommended to be performed more frequent than once per month are included in the pre-use maintenance requirements in Appendix B.

Upon request items are those which will only be required when specifically requested by operations, maintenance, or engineering personnel. Upon request items are listed in Appendix D. Most recommendations listed in Appendix A that are not in the pre-use or six month maintenance categories are categorized as 'upon request' maintenance. These are primarily requirements whose recommended frequency was greater than six months or 500 hours of operation. Because of unknowns with the LLCETS deployment schedule, it is prudent to only list these items as upon request at this time.

Appendix $E$ lists components that the vendor information states have long term storage requirements. Based upon vendor information and knowledge gained by Engineering, Operations, and Maintenance personnel from other RPP projects, long term storage is assumed to be an anticipated non-use condition for a period of at least one year. 


\section{SUMMARY}

The following are the recommended maintenance frequencies for the LLCE Transport trailer and Receiver trailer.

Appendix B, 'Pre-Use Maintenance', lists the recommended pre-use maintenance inspections required prior to field deployment. Completing the pre-use inspections will be part of the operating work package prerequisites.

Appendix C, 'Six Month Preventative Maintenance Requirements', lists the maintenance items that will be performed every six months.

Appendix D, 'Upon Request Maintenance', lists the maintenance items that the vendor recommends to have completed but does not fall within the six month or 500 hour criteria of Appendix $\mathrm{C}$, and will be performed on an as needed basis at Engineering, Operations, or Maintenance request.

Appendix E, 'Long Term Storage Maintenance Requirements', are those items required to be completed for a sustained non-operational condition of more than one year. 


\section{REFERENCES}

Accuride, RimWheel Safety \& Service Manual, Accuride Corporation

Appleton, Instruction Sheet 330086, Powerlite Series: Pin and Sleeve Receptacles. Plugs and Cable Connectors, Revision E, Appleton Electric Company 1995

Baldor, MN400, Integral Horsepower AC Induction Motors ODP Enclosure TEFC Enclosure, Baldor Motors and Drives, 1996

Control Chief, Booklet No. 8900-9000, Raymote System IR-8400 Owners Manual, Control Chief Corporation

Eagle, Maintenance Manual for Standard Hydraulic Power Units, Eagle Equipment Corporation

Hendrickson, Operator's guide HT Series Trailer Air Suspensions, Hendrickson-Turner, 1990

HNF-3252, Rev. 0, General Maintenance Instructions for LLCE Transport System, September 1998

Holland, Operating, Maintenance and Repair Procedures for the Redesigned Holland Mark $V$ Landing Gear, Holland hitch Company

Intec, Operating Guide Mode/ CVC220/220S Camera, Intec Video Systems Inc.

Intec, Operating Guide Model CVM600 Monitor, Intec Video Systems Inc

Kubota, Operator's Manual Kubota Diesel Engine, Kubota Tractor Corporation

MSI, Operation and Maintenance Manual LLCE Trailers, Mobilized Systems Inc., 1997

Nelson, Parts List and Maintenance Manual for Nelson Trailer No. 4937, Nelson Manufacturing Company

Nelson, Parts List and Maintenance Manual for Nelson Trailer No. 4938, Nelson Manufacturing Company

Stamford, Publication No. BCH-018, Installation, Service \& Maintenance Manual for BC Range of Generators, $6^{\text {th }}$ Ed., Newage Limited

Thern, Owner's Manual for Worm/Spur Gear Series 4WS Power Winches, Thern Incorporated

Webb, Bulletin IM-989, Installation, Service and Safety Instructions, Revision 1, Webb Wheel Products Incorporated 


\section{GENERAL VENDOR PREVENTATIVE MAINTENANCE}

\begin{tabular}{|c|c|c|}
\hline Gomponentpert & Haintenonce Acron & 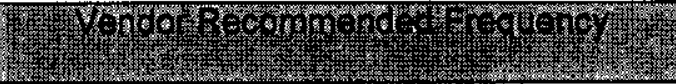 \\
\hline \multirow[t]{21}{*}{ Diesel Engine } & Check engine coolant level. & Every 8 hours during operation. \\
\hline & Charge battery. & $\begin{array}{l}\text { Monthly during summer and every } 2 \\
\text { months during winter. }\end{array}$ \\
\hline & Replace the air cleaner filter element. & Yearly or every 6 cleanings. \\
\hline & Replace battery. & \multirow[t]{4}{*}{ Every 2 years. } \\
\hline & $\begin{array}{l}\text { Replace fuel pipes and clamp bands. } \\
\text { Bleed system. }\end{array}$ & \\
\hline & $\begin{array}{l}\text { Replace radiator hoses and clamp } \\
\text { bands. }\end{array}$ & \\
\hline & Replace engine coolant. & \\
\hline & Change engine oil. & $\begin{array}{l}\text { After first } 50 \text { hours of operation and every } \\
150 \text { hours of operation thereafter. }\end{array}$ \\
\hline & Replace the oil filter cartridge. & $\begin{array}{l}\text { After first } 50 \text { hours of operation and every } \\
300 \text { hours of operation thereafter. }\end{array}$ \\
\hline & $\begin{array}{l}\text { Inspect/replace/tighten fuel pipes and } \\
\text { clamp bands. }\end{array}$ & $\begin{array}{l}\text { Every } 50 \text { hours of operation or every } 6 \\
\text { months. }\end{array}$ \\
\hline & $\begin{array}{l}\text { Inspect/clean the air cleaner filter } \\
\text { element and housing. }\end{array}$ & \multirow[t]{4}{*}{ Every 100 hours of operation. } \\
\hline & Check battery electrolyte level. & \\
\hline & Clean fuel filter. & \\
\hline & Check fan belt. & \\
\hline & $\begin{array}{l}\text { Inspect radiator hoses and clamp } \\
\text { bands. }\end{array}$ & $\begin{array}{l}\text { Every } 200 \text { hours of operation or every } 6 \\
\text { months. }\end{array}$ \\
\hline & Replace fuel filter cartridge. Vent air. & Every 400 hours of operation. \\
\hline & Replace fan belt. & \multirow[t]{2}{*}{ Every 500 hours of operation. } \\
\hline & Clean engine cooling system. & \\
\hline & $\begin{array}{l}\text { Inspect/Remove sediment from fuel } \\
\text { tank. }\end{array}$ & Every 500 hours of operation. \\
\hline & Check engine valve clearance. & Every 800 hours of operation. \\
\hline & Run engine for 5 minutes with no leaks. & $\begin{array}{l}\text { Every } 2-3 \text { months during periods when the } \\
\text { engine is not being operated. }\end{array}$ \\
\hline \multirow[t]{4}{*}{ Generator } & Inspect/clean air filters. & $\begin{array}{l}\text { Every } 100 \text { hours of operation or every } 3 \\
\text { months. }\end{array}$ \\
\hline & Inspect/clean generator interior/exterior. & \multirow{2}{*}{$\begin{array}{l}\text { Every } 500 \text { hours of operation or every } 3 \\
\text { months. }\end{array}$} \\
\hline & Check insulation resistance of windings. & \\
\hline & Replace generator bearings. & Every 40,000 hours of operation. \\
\hline
\end{tabular}


APPENDIX A

Hydraulic System Preventative Maintenance

\begin{tabular}{|c|c|c|}
\hline Componenvearty & Wainterence Actoris & 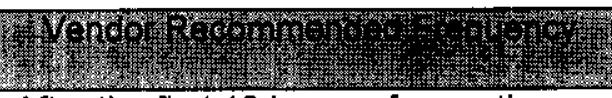 \\
\hline \multirow[t]{14}{*}{ Hydraulic System } & Check suction strainers. & $\begin{array}{l}\text { After the first } 10 \text { hours of operation } \\
\text { and every } 100 \text { hours thereafter. }\end{array}$ \\
\hline & Check level of hydraulic fluid. & Every 100 hours of operation \\
\hline & Check reservoir heater and thermostat & $\begin{array}{l}\text { Every } 500 \text { hours of operation or every } \\
6 \text { months. }\end{array}$ \\
\hline & Replace hydraulic fluid. & Every $1,000-2,000$ hours of operation. \\
\hline & Visual inspection of pump motors. & \multirow{3}{*}{$\begin{array}{l}\text { Every } 500 \text { hours of operation or every } \\
3 \text { months. }\end{array}$} \\
\hline & $\begin{array}{l}\text { Check insulation resistance of pump } \\
\text { motor windings. }\end{array}$ & \\
\hline & $\begin{array}{l}\text { Check tightness of electrical } \\
\text { connections. }\end{array}$ & \\
\hline & Clean hydraulic system components. & \multirow[t]{6}{*}{ Every 6 months. } \\
\hline & $\begin{array}{l}\text { Sample and visually check hydraulic } \\
\text { fluid. }\end{array}$ & \\
\hline & $\begin{array}{l}\text { Inspect reservoir heater and } \\
\text { thermostat. }\end{array}$ & \\
\hline & $\begin{array}{l}\text { Check motor cleanliness and } \\
\text { vent/drain openings. }\end{array}$ & \\
\hline & $\begin{array}{l}\text { Check tightness of electrical connects } \\
\text { on motor. }\end{array}$ & \\
\hline & Check operation of all manual valves. & \\
\hline & Clean/check/replace fluid filters & $\begin{array}{l}\text { As indicated by CLOGGED FILTER } \\
\text { alarm }\end{array}$ \\
\hline $\begin{array}{l}\text { Hydraulic Cylinders } \\
\text { (Transport Trailer strap } \\
\text { latch mechanism) }\end{array}$ & Check rod seals for leakage. & Every 12 months \\
\hline
\end{tabular}

Electrical and Control Systems Preventative Maintenance

\begin{tabular}{|c|c|c|}
\hline Componentertits & 1) & 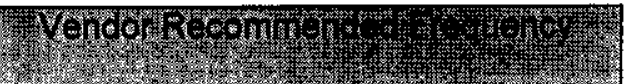 \\
\hline IR Remote Controls & Charge batteries. & $\begin{array}{l}\text { Every } 8 \text { hours of operation (when } \\
\text { discharged). }\end{array}$ \\
\hline $\begin{array}{l}\text { Enclosure Heat } \\
\text { Exchangers }\end{array}$ & Inspect and clean air filters. & Monthly during operation. \\
\hline $\begin{array}{l}\text { Electrical Components } \\
\text { and Enclosures }\end{array}$ & $\begin{array}{l}\text { Inspect and clean electrical } \\
\text { components, connections, and wiring. }\end{array}$ & \multirow[t]{3}{*}{ Every 6 months during operation. } \\
\hline Circuit Breakers & Check operation. & \\
\hline Cable Reel & Lubricate. & \\
\hline Shore Power Cable & $\begin{array}{l}\text { Inspect cable, plugs, connectors, and } \\
\text { receptacles. }\end{array}$ & Yearly during operation. \\
\hline
\end{tabular}


APPENDIX A

Slide and Outrigger Preventative Maintenance

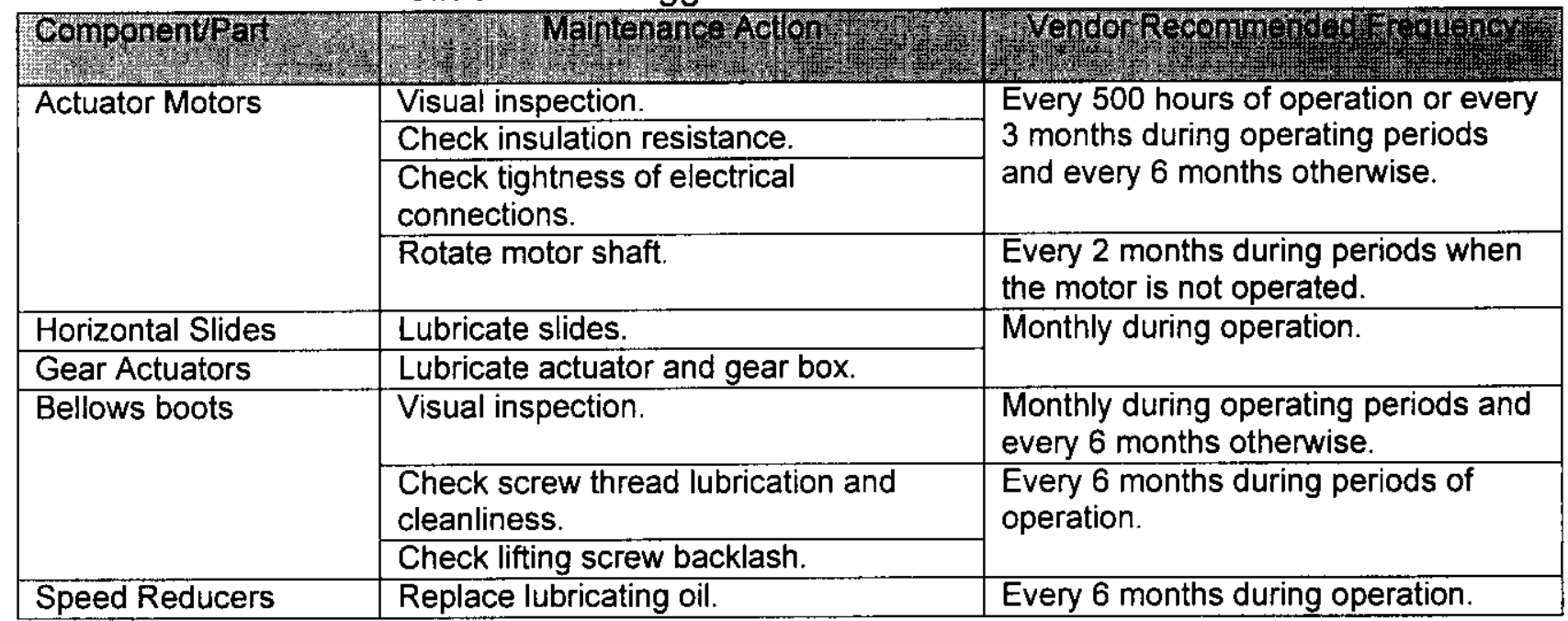

\section{Strongback Preventative Maintenance}

\begin{tabular}{|c|c|c|}
\hline \multicolumn{2}{|c|}{ ComponenvPart } & Yopdor Recomand \\
\hline $\begin{array}{l}\text { Skid Lower Guide } \\
\text { Wheels }\end{array}$ & Lubricate. & \multirow[t]{2}{*}{ Monthly during operation. } \\
\hline Latch Mechanism & Lubricate. & \\
\hline \multirow[t]{2}{*}{ Latch Motors } & Visual inspection. & \multirow{3}{*}{$\begin{array}{l}\text { Every } 500 \text { hours of operation or every } \\
3 \text { months (during periods operation }\end{array}$} \\
\hline & Check insulation resistance. & \\
\hline $\begin{array}{l}\text { Strongback Electrical } \\
\text { Assembly }\end{array}$ & $\begin{array}{l}\text { Inspect electrical connections and } \\
\text { wiring. }\end{array}$ & \\
\hline
\end{tabular}

\section{Trailer Preventative Maintenance}

\begin{tabular}{|c|c|c|}
\hline Componenupart & W Maineriance Action, & 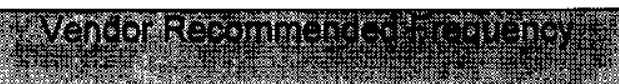 \\
\hline \multirow[t]{2}{*}{ Tires } & Visual inspection. & \multirow[t]{3}{*}{ Daily during operation } \\
\hline & Check air pressure. & \\
\hline \multirow[t]{3}{*}{ Hubs } & Visual inspection. & \\
\hline & Check capnut and/or flange nut torque. & $\begin{array}{l}\text { After first } 50-100 \text { miles and every } \\
2000-4000 \text { miles thereafter. }\end{array}$ \\
\hline & Replace bearing lubricant. & Every 25,000 miles. \\
\hline \multirow[t]{2}{*}{ Wheels } & Visual inspection. & Daily during operation. \\
\hline & Check rim nut torque. & $\begin{array}{l}\text { After first } 50-100 \text { miles and every } \\
2,000-4,000 \text { miles thereafter. }\end{array}$ \\
\hline $\begin{array}{l}\text { Undercarriage Lock } \\
\text { Mechanism }\end{array}$ & \multirow[t]{2}{*}{ Lubricate. } & \multirow[t]{2}{*}{$\begin{array}{l}\text { Lubricate every } 6 \text { months during } \\
\text { operation. }\end{array}$} \\
\hline $\begin{array}{l}\text { Landing Gear (trailers } \\
\text { and personnel access } \\
\text { platform) }\end{array}$ & & \\
\hline
\end{tabular}


APPENDIX A

Tug Preventative Maintenance

\begin{tabular}{|c|c|c|}
\hline Componenterit & Taintenance Action & Vencor Recommended Frouency \\
\hline \multirow[t]{4}{*}{ Actuator Motor } & Visual inspection. & \multirow{3}{*}{$\begin{array}{l}\text { Every } 500 \text { hours of operation or every } \\
3 \text { months during operating periods } \\
\text { and every } 6 \text { months otherwise. }\end{array}$} \\
\hline & Check insulation resistance. & \\
\hline & $\begin{array}{l}\text { Check tightness of electrical } \\
\text { connections. }\end{array}$ & \\
\hline & Rotate motor shaft. & $\begin{array}{l}\text { Every } 2 \text { months during periods when } \\
\text { the motor is not operated. }\end{array}$ \\
\hline Tug Wheels & Lubricate. & \multirow[t]{2}{*}{ Monthly during operation. } \\
\hline Gear Actuator & Lubricate actuator and gear box. & \\
\hline \multirow[t]{3}{*}{ Bellows Boots } & Visual inspection. & $\begin{array}{l}\text { Monthly during operating periods and } \\
\text { every } 6 \text { months otherwise. }\end{array}$ \\
\hline & $\begin{array}{l}\text { Check screw thread lubrication and } \\
\text { cleanliness. }\end{array}$ & \multirow[t]{2}{*}{$\begin{array}{l}\text { Every } 6 \text { months during operating } \\
\text { periods. }\end{array}$} \\
\hline & Check screw backlash. & \\
\hline Speed Reducer & Replace lubricating oil. & Every 6 months during operation. \\
\hline
\end{tabular}

Tug Winch Preventative Maintenance

\begin{tabular}{|c|c|c|}
\hline Componenteren & 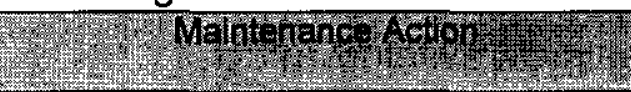 & 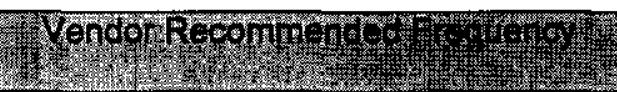 \\
\hline \multirow[t]{6}{*}{ Winch Assembly } & Detailed inspection. & \multirow{2}{*}{$\begin{array}{l}\text { After the first } 10 \text { hours of operation } \\
\text { and every } 6 \text { months during periods of } \\
\text { operation. }\end{array}$} \\
\hline & Check electrical connections and wiring. & \\
\hline & Check winch drum. & \multirow{3}{*}{$\begin{array}{l}\text { Every } 6 \text { months during operating } \\
\text { periods. }\end{array}$} \\
\hline & Check winch foundation. & \\
\hline & Load test at $10 \%$ design load. & \\
\hline & Clean winch assembly components. & Every 6 months \\
\hline \multirow[t]{3}{*}{ Winch } & $\begin{array}{l}\text { Sample and visually check reducer } \\
\text { lubricant. }\end{array}$ & $\begin{array}{l}\text { After first } 5 \text { hours of operation and } \\
\text { every } 6 \text { months during periods of } \\
\text { operation. }\end{array}$ \\
\hline & Rotate winch drum. & $\begin{array}{l}\text { Every } 2 \text { months during periods when } \\
\text { the winch is not operated. }\end{array}$ \\
\hline & Replace reducer lubricant. & Every 2 years \\
\hline \multirow[t]{2}{*}{ Motor } & Visual inspection. & \multirow{2}{*}{$\begin{array}{l}\text { After the first } 10 \text { hours of operation } \\
\text { and every } 500 \text { hours of operation or } \\
\text { every } 3 \text { months during periods of } \\
\text { operation. }\end{array}$} \\
\hline & Check insulation resistance. & \\
\hline \multirow[t]{2}{*}{ Wire Rope } & Visual inspection. & \multirow{2}{*}{$\begin{array}{l}\text { According to Section } 8.0 \text { of the } \\
\text { Hanford Site Hoisting and Rigging } \\
\text { Manual. }\end{array}$} \\
\hline & Lubricate sheaves. & \\
\hline
\end{tabular}




\section{APPENDIX B}

\section{PRE-USE MAINTENANCE}

\section{Diesel Generator Pre-Use Inspection}

\begin{tabular}{|l|l|}
\hline Componentreart & \\
\hline \multirow{5}{*}{ Diesel Engine } & $\begin{array}{l}\text { Visual Inspection. Check for overall condition and damaged, loose or missing } \\
\text { parts. }\end{array}$ \\
\hline & Check engine coolant level. \\
\cline { 2 - 2 } & Check engine fuel level. \\
\cline { 2 - 2 } & Check engine oil level. \\
\cline { 2 - 2 } & $\begin{array}{l}\text { Inspect the air cleaner filter element, filter housing, and cap. Clean, if } \\
\text { necessary. }\end{array}$ \\
\cline { 2 - 2 } & Check battery electrolyte level and charge. \\
\hline Generator & Inspect air filters. Clean and charge if necessary. \\
\hline
\end{tabular}

Electrical and Control Systems Pre-Use Maintenance

\begin{tabular}{|l|l|}
\hline ComponentPart & \multicolumn{1}{|c|}{ 19inionance Action } \\
\hline IR Remote Controls & Inspect/Recharge/Replace batteries. \\
\hline $\begin{array}{l}\text { Enclosure Heat } \\
\text { Exchanges }\end{array}$ & Inspect and clean filters. \\
\hline Electrical Components & $\begin{array}{l}\text { Check and replace light bulbs if necessary. } \\
\text { Check operability of cameras. }\end{array}$ \\
\hline
\end{tabular}

Trailer Pre-Use Maintenance

\begin{tabular}{|l|l|}
\hline ComponentPart & \multicolumn{1}{|c|}{ Tre Mainonance. Acton } \\
\hline \multirow{2}{*}{ Tires } & Check tire pressure. \\
\cline { 2 - 2 } & Visual inspection. \\
\hline $\begin{array}{l}\text { Hubs, Wheels, and } \\
\text { Undercarriage }\end{array}$ & Visual inspection. \\
\hline
\end{tabular}




\section{APPENDIX B}

\section{Tug Pre-Use Maintenance}

\begin{tabular}{|l|l|}
\hline ComponenuPart & \multicolumn{1}{|c|}{ Mainenance Action } \\
\hline Tug Wheels & Check and clean wheel track. \\
\hline Bellows Boots & Visual inspection. \\
\hline Speed Reducer & Check breather plug installed. \\
\hline
\end{tabular}

Tug Winch Pre-Use Maintenance

\begin{tabular}{|l|l|}
\hline ComponentPar & \multicolumn{1}{|c|}{ Malntenahce Action } \\
\hline \multirow{3}{*}{ Winch Assembly } & Visual inspection. \\
\cline { 2 - 2 } & Check reducer lubricant level. \\
\hline \multirow{3}{*}{ Winch } & Lubricate spur gears. \\
\cline { 2 - 2 } & Lubricate flange bearings. \\
\cline { 2 - 2 } & Check reducer lubricant level. \\
\hline Motor & Check motor cleanliness. \\
\hline Wire Rope & Pre-use maintenance according to Hanford Site Rigging and Hoisting Manual. \\
\hline
\end{tabular}


RPP-5696, Rev. 0

APPENDIX C

SIX MONTH PREVENTATIVE MAINTENANCE REQUIREMENTS

Diesel Generator 6 Mo. Maintenance

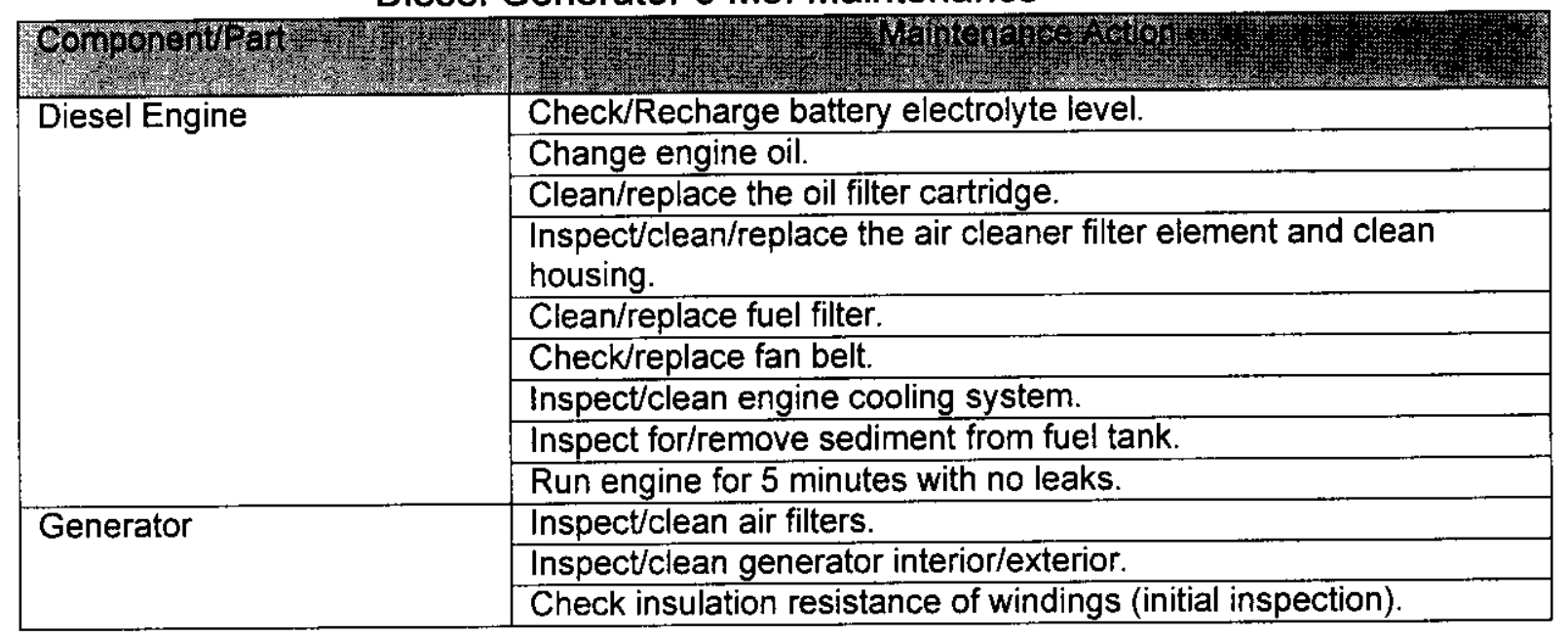

Hydraulic System 6 Mo. Maintenance

\begin{tabular}{|c|c|}
\hline \multirow{2}{*}{\multicolumn{2}{|c|}{\begin{tabular}{|l|l} 
Componenppart & \\
Hydraulic System & Check suction strainers. \\
\end{tabular}}} \\
\hline \multirow[t]{12}{*}{ Hydraulic System } & \\
\hline & Check level of hydraulic fluid. \\
\hline & Check reservoir heater and thermostat \\
\hline & Visual inspection of pump motors. \\
\hline & $\begin{array}{l}\text { Check insulation resistance of pump motor windings (initial } \\
\text { inspection). }\end{array}$ \\
\hline & Check tightness of electrical connections. \\
\hline & Clean hydraulic system components. \\
\hline & Sample and visually check hydraulic fluid. \\
\hline & Check motor cleanliness and vent/drain openings. \\
\hline & Check tightness of electrical connects on motor. \\
\hline & Check operation of all manual valves. \\
\hline & Clean/check/replace fluid filters \\
\hline $\begin{array}{l}\text { Hydraulic Cylinders } \\
\text { (strongback cylinders, strap } \\
\text { latch mechanism, trailer } \\
\text { bogeys) }\end{array}$ & Check rod cylinders for leakage. \\
\hline
\end{tabular}




\section{APPENDIX C}

Electrical and Control Systems 6 Mo. Maintenance

\begin{tabular}{|l|l|}
\hline Componentean & \\
\hline Enclosure Heat Exchangers & Inspect and clean air filters. \\
\hline $\begin{array}{l}\text { Electrical Components } \\
-\quad \text { lights }\end{array}$ & $\begin{array}{l}\text { Inspect/tighten and clean/replace electrical components, } \\
\text { connections, and wiring. }\end{array}$ \\
$-\quad$ limit switches \\
$-\quad$ electrical cabinet, etc. & \\
\hline Circuit Breakers & Check operation. \\
\hline Cable Reel & Lubricate. \\
\hline Shore Power Cable & Inspect cable, plugs, connectors, and receptacles. \\
\hline
\end{tabular}

Slide and Outrigger 6 Mo. Maintenance

\begin{tabular}{|l|l|}
\hline Componenuear & \\
\hline \multirow{5}{*}{ Actuator Motors } & Visual inspection. \\
\cline { 2 - 2 } & Check insulation resistance (initial inspection). \\
\cline { 2 - 2 } & Check tightness of electrical connections. \\
\cline { 2 - 2 } & Rotate motor shaft. \\
\hline Horizontal Slides & Lubricate slides. \\
\hline Gear Actuators & Lubricate actuator and gearbox. \\
\hline Bellows boots & Visual inspection. \\
\cline { 2 - 2 } & Check screw thread lubrication and cleanliness. \\
\cline { 2 - 2 } & Check lifting screw backlash. \\
\hline Speed Reducers & Inspect/replace lubricating oil. \\
\hline
\end{tabular}

Strongback Preventative 6 Mo. Maintenance

\begin{tabular}{|l|l|}
\hline ComponenwPart & \\
\hline Skid Lower Guide Wheels & Lubricate. \\
\hline Latch Mechanism & Lubricate. \\
\hline Latch Motors & Visual inspection. \\
\cline { 2 - 2 } & Check insulation resistance (initial inspection). \\
\hline $\begin{array}{l}\text { Strongback Electrical } \\
\text { Assembly }\end{array}$ & Inspect electrical connections and wiring. \\
\hline
\end{tabular}

Trailer Preventative 6 Mo. Maintenance

\begin{tabular}{|l|l|}
\hline \multirow{2}{*}{ Componenpart } & \\
\hline \multirow{2}{*}{ Tires } & Visual inspection. \\
\cline { 2 - 2 } & Check air pressure. \\
\hline Hubs & Visual inspection. \\
\hline Wheels & Visual inspection. \\
\hline $\begin{array}{l}\text { Undercarriage Lock } \\
\text { Mechanism }\end{array}$ & Lubricate as required. \\
\hline $\begin{array}{l}\text { Landing Gear (trailers and } \\
\text { personnel access platform) }\end{array}$ & \\
\hline
\end{tabular}


RPP-5696, Rev. 0

APPENDIX C

Tug Preventative 6 Mo. Maintenance

\begin{tabular}{|c|c|}
\hline Componenveary & 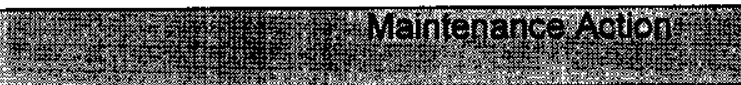 \\
\hline \multirow[t]{4}{*}{ Actuator Motor } & Visual inspection. \\
\hline & Check insulation resistance (initial inspection). \\
\hline & Check tightness of electrical connections. \\
\hline & Rotate motor shaft. \\
\hline Tug Wheels & Lubricate. \\
\hline Gear Actuator & Lubricate actuator and gearbox. \\
\hline \multirow{3}{*}{ Bellows Boots } & Visual inspection. \\
\hline & Check screw thread lubrication and cleanliness. \\
\hline & Check screw backlash. \\
\hline Speed Reducer & Replace lubricating oil. \\
\hline
\end{tabular}

Tug Winch 6 Mo. Maintenance

\begin{tabular}{|c|c|}
\hline Componentpart & 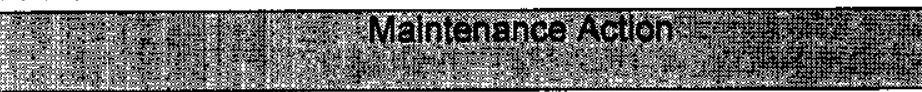 \\
\hline \multirow{5}{*}{$\begin{array}{l}\text { Winch Assembly (per } \\
\text { applicable Hanford Hoisting } \\
\text { and Rigging manual) }\end{array}$} & Check electrical connections and wiring. \\
\hline & Check winch drum. \\
\hline & Check winch foundation. \\
\hline & $\begin{array}{l}\text { Load test at } 10 \% \text { rated capacity, if required by Hoisting and } \\
\text { Rigging manual. }\end{array}$ \\
\hline & Clean winch assembly components. \\
\hline \multirow{2}{*}{$\begin{array}{l}\text { Winch Assembly (per } \\
\text { applicable Hanford Hoisting } \\
\text { and Rigging manual) }\end{array}$} & Sample and visually check reducer lubricant. \\
\hline & Rotate winch drum. \\
\hline \multirow[t]{2}{*}{ Motor } & Visual inspection. \\
\hline & Check insulation resistance (initial inspection). \\
\hline \multirow[t]{2}{*}{ Wire Rope } & Visual inspection. \\
\hline & Lubricate sheaves. \\
\hline
\end{tabular}


APPENDIX D

UPON REQUEST MAINTENANCE

Diesel Generator Recall Maintenance

\begin{tabular}{|l|l|}
\hline Componentpartis & \\
\hline \multirow{4}{*}{ Diesel Engine } & Replace battery. \\
\hline \multirow{5}{*}{} & Replace fuel pipes and clamp bands. Bleed system. \\
\cline { 2 - 2 } & Replace radiator hoses and clamp bands. \\
\cline { 2 - 2 } & Replace engine coolant. \\
\cline { 2 - 2 } & Check engine valve clearance. \\
\cline { 2 - 2 } & Run engine for 5 minutes with no leaks. \\
\hline Generator & Replace generator bearings. \\
\hline
\end{tabular}

Hydraulic System Recall Maintenance

\begin{tabular}{|c|c|}
\hline Hydraulic System & Replace hydraulic fluid. \\
\hline
\end{tabular}

Trailer Recall Maintenance

\begin{tabular}{|l|l|}
\hline $\begin{array}{l}\text { ComponentPait } \\
\text { Hubs }\end{array}$ & \multicolumn{1}{|c|}{} \\
\hline \multirow{2}{*}{ Wheels } & Check capnut and/or flange nut torque. \\
\cline { 2 - 2 } & Replace bearing lubricant. \\
\hline
\end{tabular}

Tug Winch Recall Maintenance

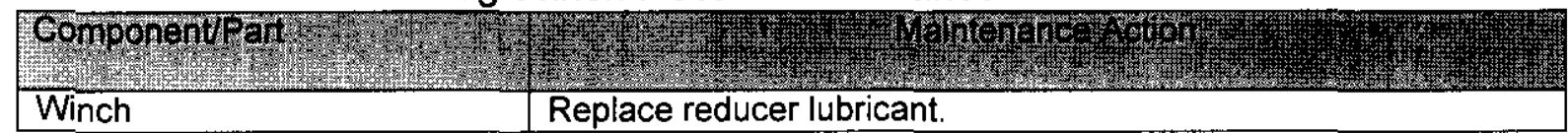


RPP-5696, Rev. 0

APPENDIX E

\section{LONG TERM STORAGE MAINTENANCE REQUIREMENTS}

Diesel Generator Preventative Maintenance

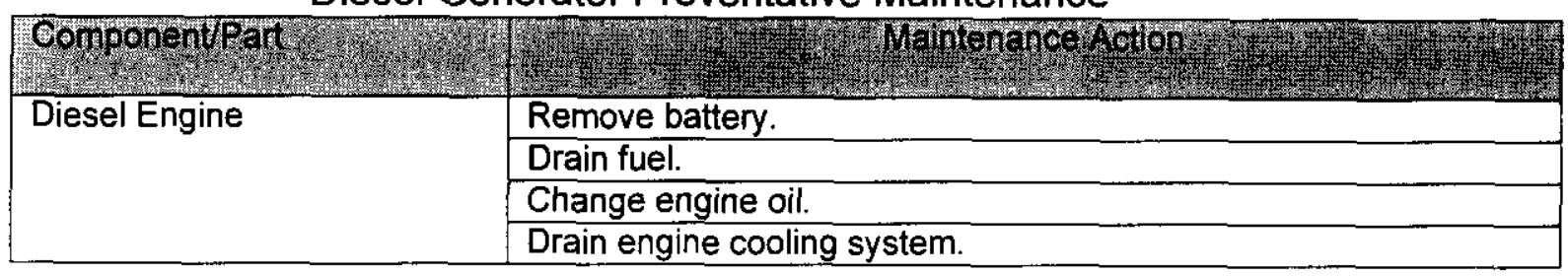

